\title{
Corrections
}

\section{Correction: Ainsworth et al., Dual Gamma Rhythm Generators Control Interlaminar Synchrony in Auditory} Cortex

In the article "Dual Gamma Rhythm Generators Control Interlaminar Synchrony in Auditory Cortex" by Matthew Ainsworth, Shane Lee, Mark O. Cunningham, Anita K. Roopun, Roger D. Traub, Nancy J. Kopell, and Miles A. Whittington, which appeared on pages 17040-17051 of the November 23, 2011 issue, the authors regret that two of the authors (S.L, N.J.K.) were omitted from one section of the author contribution footnote. The footnote should have read "Author contributions: M.A.W. designed research; M.A., S.L., and A.K.R. performed research; M.O.C., R.D.T., N.J.K., and M.A.W. analyzed data; M.A.W., S.L., and N.J.K. wrote the paper.”

DOI: 10.1523/JNEUROSCI.6433-11.2011 